\title{
Web Tables
}

\section{Web Table 1}

Mean and standard deviation (SD) of p-values in simulation results under equal missing rates $(15 \%, 15 \%)$

\begin{tabular}{|c|c|c|c|c|c|c|c|c|c|c|c|}
\hline \multirow{3}{*}{ Scenarios } & \multirow[b]{3}{*}{$\left(\mu_{1}, \mu_{2}\right)$} & & & \multicolumn{4}{|c|}{ Ignorable missingness } & \multicolumn{4}{|c|}{ Nonignorable missingness } \\
\hline & & \multicolumn{2}{|c|}{$\mathrm{CC}$} & \multicolumn{2}{|c|}{$T_{W}$} & \multicolumn{2}{|c|}{$T_{L R}$} & \multicolumn{2}{|c|}{$T_{W}$} & \multicolumn{2}{|c|}{$T_{L R}$} \\
\hline & & Mean & $\mathrm{SD}$ & Mean & $\mathrm{SD}$ & Mean & $\mathrm{SD}$ & Mean & $\mathrm{SD}$ & Mean & $\mathrm{SD}$ \\
\hline \multicolumn{12}{|c|}{ Substitution method } \\
\hline$M N A R$-mlo & $(0,0)$ & 0.66 & 0.01 & 0.66 & 0.02 & 0.66 & 0.02 & 0.51 & 0.19 & 0.50 & 0.19 \\
\hline$M N A R-m l o$ & $(0.5,1)$ & 0.47 & 0.15 & 0.58 & 0.16 & 0.58 & 0.16 & 0.18 & 0.19 & 0.17 & 0.19 \\
\hline$M N A R-m l o$ & $(1,2)$ & 0.36 & 0.19 & 0.20 & 0.21 & 0.20 & 0.20 & 0.02 & 0.05 & 0.02 & 0.05 \\
\hline$M N A R-m s o$ & $(0,0)$ & 0.07 & 0.12 & 0.16 & 0.19 & 0.16 & 0.19 & 0.42 & 0.23 & 0.42 & 0.23 \\
\hline$M N A R$-mso & $(-1,-1)$ & 0.25 & 0.19 & 0.52 & 0.19 & 0.52 & 0.19 & 0.60 & 0.14 & 0.60 & 0.14 \\
\hline$M A R-l o$ & $(0.5,1)$ & 0.33 & 0.19 & 0.15 & 0.18 & 0.15 & 0.18 & 0.03 & 0.06 & 0.02 & 0.06 \\
\hline$M A R$-so & $(0,0)$ & 0.06 & 0.10 & 0.43 & 0.22 & 0.43 & 0.22 & 0.54 & 0.18 & 0.54 & 0.18 \\
\hline$M C A R$ & $(0,0)$ & 0.32 & 0.19 & 0.43 & 0.22 & 0.43 & 0.22 & 0.44 & 0.23 & 0.44 & 0.23 \\
\hline$M C A R$ & $(1,2)$ & 0.02 & 0.06 & 0.01 & 0.04 & 0.01 & 0.04 & 0.03 & 0.07 & 0.03 & 0.07 \\
\hline \multicolumn{12}{|c|}{ Bound method $^{a}$} \\
\hline$M N A R-m l o$ & $(0,0)$ & 1.00 & 0.00 & 1.00 & 0.03 & 1.00 & 0.03 & 0.81 & 0.27 & 0.81 & 0.28 \\
\hline$M N A R-m l o$ & $(0.5,1)$ & 0.99 & 0.05 & 0.89 & 0.22 & 0.89 & 0.22 & 0.31 & 0.31 & 0.31 & 0.31 \\
\hline$M N A R-m l o$ & $(1,2)$ & 0.80 & 0.28 & 0.33 & 0.32 & 0.33 & 0.32 & 0.03 & 0.08 & 0.03 & 0.10 \\
\hline$M N A R-m s o$ & $(0,0)$ & 0.14 & 0.21 & 0.28 & 0.30 & 0.29 & 0.30 & 0.67 & 0.33 & 0.67 & 0.33 \\
\hline$M N A R-m s o$ & $(-1,-1)$ & 0.56 & 0.35 & 0.82 & 0.27 & 0.82 & 0.27 & 0.92 & 0.18 & 0.92 & 0.19 \\
\hline$M A R-l o$ & $(0.5,1)$ & 0.76 & 0.30 & 0.27 & 0.29 & 0.26 & 0.29 & 0.05 & 0.11 & 0.05 & 0.11 \\
\hline$M A R-s o$ & $(0,0)$ & 0.11 & 0.18 & 0.70 & 0.32 & 0.70 & 0.32 & 0.84 & 0.26 & 0.84 & 0.26 \\
\hline$M C A R$ & $(0,0)$ & 0.72 & 0.32 & 0.70 & 0.32 & 0.70 & 0.32 & 0.68 & 0.32 & 0.68 & 0.33 \\
\hline$M C A R$ & $(1,2)$ & 0.05 & 0.11 & 0.03 & 0.07 & 0.03 & 0.07 & 0.05 & 0.12 & 0.05 & 0.12 \\
\hline
\end{tabular}

${ }^{a}$ Mean and SD are for the upper bound of p-values. 


\section{Web Table 2}

Empirical Type I error rates and power in simulation results under equal

\begin{tabular}{|c|c|c|c|c|c|c|}
\hline \multirow[b]{2}{*}{ Scenarios } & \multirow[b]{2}{*}{$\left(\mu_{1}, \mu_{2}\right)$} & \multirow[b]{2}{*}{$\mathrm{CC}$} & \multicolumn{2}{|c|}{ Ignorable missingness } & \multicolumn{2}{|c|}{ Nonignorable missingness } \\
\hline & & & $T_{W}$ & $T_{L R}$ & $T_{W}$ & $T_{L R}$ \\
\hline \multicolumn{7}{|c|}{ Substitution method, Type I error rates (in \%) } \\
\hline$M N A R-m l o$ & $(0,0)$ & 0.0 & 0.0 & 0.0 & 2.0 & 2.3 \\
\hline$M N A R$-mso & $(0,0)$ & 64 & 42 & 41 & 6.4 & 6.2 \\
\hline$M N A R-m s o$ & $(-1,-1)$ & 19 & 2.1 & 2.1 & 0.4 & 0.5 \\
\hline$M A R$-so & $(0,0)$ & 71 & 5.0 & 4.8 & 2.1 & 1.9 \\
\hline$M C A R$ & $(0,0)$ & 8.3 & 5.1 & 5.0 & 5.7 & 6.0 \\
\hline \multicolumn{7}{|c|}{ Bound method, Type I error rates (in \%) } \\
\hline MNAR-mlo & $(0,0)$ & 0.0 & 0.0 & 0.0 & 1.0 & 1.1 \\
\hline$M N A R$-mso & $(0,0)$ & 51 & 30 & 29 & 3.5 & 3.3 \\
\hline MNAR-mso & $(-1,-1)$ & 8.0 & 0.8 & 0.8 & 0.2 & 0.5 \\
\hline$M A R-s o$ & $(0,0)$ & 58 & 2.6 & 2.5 & 0.9 & 1.0 \\
\hline$M C A R$ & $(0,0)$ & 2.6 & 2.8 & 2.7 & 2.7 & 2.9 \\
\hline \multicolumn{7}{|c|}{ Substitution method, power (in \%) } \\
\hline MNAR-mlo & $(0.5,1)$ & 0.0 & 1.0 & 1.0 & 36 & 38 \\
\hline MNAR-mlo & $(1,2)$ & 4.8 & 35 & 35 & 92 & 92 \\
\hline MNAR-mso & $(0.5,0.5)$ & 88 & 74 & 74 & 21 & 19 \\
\hline$M A R-l o$ & $(0.5,1)$ & 7.6 & 43 & 44 & 86 & 87 \\
\hline$M C A R$ & $(1,2)$ & 87 & 94 & 94 & 87 & 86 \\
\hline \multicolumn{7}{|c|}{ Bound method, power (in \%) } \\
\hline$M N A R$-mlo & $(0.5,1)$ & 0.0 & 0.4 & 0.4 & 24 & 26 \\
\hline MNAR-mlo & $(1,2)$ & 1.0 & 24 & 24 & 85 & 86 \\
\hline$M N A R$-mso & $(0.5,0.5)$ & 79 & 63 & 61 & 13 & 12 \\
\hline$M A R-l o$ & $(0.5,1)$ & 1.8 & 31 & 32 & 78 & 79 \\
\hline$M C A R$ & $(1,2)$ & 77 & 88 & 88 & 82 & 80 \\
\hline
\end{tabular}

